\title{
A study of SSPE: early clinical features
}

\author{
Satish V. Khadilkar, Shekhar G. Patil, Kedar S. Kulkarni
}

Department of Neurology, Grant Medical College and Sir JJ Group of Hospitals, Mumbai, India

\begin{abstract}
Thirty two patients with subacute sclerosing panencephalitis (SSPE) admitted under the care of Department of Neurology at JJ Hospital and Grant Medical College, Mumbai during the period 1998-2003 were analyzed. All patients were evaluated clinically, with relevant investigations and neuroimaging wherever possible. Particular attention was given to early clinical features. Diagnosis was confirmed by cerebrospinal fluid study for measles antibody and by electroencephalography. The mean age of our patients was $\mathbf{1 3 . 4}$ years and the vaccinated patients tended to be older. Nine patients had received measles vaccination. Twelve percent of patients were older than the age of 20 years at the onset of symptoms. Approximately 40.6\% of patients presented with symptoms of loss of vision, seizures and behavioral change. At this stage myoclonus and cognitive decline were conspicuous by their absence. Eventually typical features like myoclonus and cognitive decline evolved after a mean period of 8 months. Even in the present era, SSPE continues to remain the most important cause of progressive myoclonic epilepsy. With progressive increase in age of presentation, in patients with features like loss of vision, seizures and behavioral changes, SSPE should be carefully considered. (J Pediatr Neurol 2004; 2(2): 73-77).
\end{abstract}

Key words: early clinical features, SSPE, vision loss, seizures.

Correspondence: Dr. Satish V Khadilkar

Room no 110, First Floor,

MRC Bulding,

Bombay Hospital, Mumbai, India.

E-mail: khadilkar@vsnl.com

Received: November 02, 2003

Revised: December 24, 2003.

Accepted: January 16, 2004.

\section{Introduction}

Subacute sclerosing panencephalitis (SSPE) is a slowly progressive fatal inflammatory disease of the central nervous system, developing as a sequel to childhood measles infection $(1,2)$. Typically it presents with myoclonus and dementia progressing to a mute, bed-ridden and incontinent state finally leading to death. The best accepted postulation is that during the measles infection there is incomplete clearance of the measles virus by the patient's immune system leading to persistence of incomplete forms of the virus with aberrant $M$ protein in the central nervous system. This results in cell death, inflammation and gliosis (1).

The worldwide prevalence of SSPE is $0.04-2$ cases per million $(1,3)$. It has been brought down following implementation of measles vaccination in the developed countries (4). SSPE still exists in the developing nations, with high incidence amongst some ethnic groups $(3,6)$. The pattern of clinical presentation of SSPE has been noticed to change to an extent over the years, while some changes have also been noted in the laboratory features of SSPE $(1,2)$. SSPE still continues to take lives of children in developing countries (6); the uncommon modes of presentation of SSPE pose diagnostic difficulties and hence are being highlighted in this study. We have also compared the present results with a similar study carried out in the same department of neurology in 1974 (7).

\section{Materials and Methods}

This study was carried out in the department of neurology of a tertiary care hospital during the period of five years from 1998 to 2003.

Inclusion criteria given by Dyken (1) were followed, namely:

1. Progressive cognitive decline with stereotyped myoclonic jerks.

2. Generalized long-interval periodic complexes in the electroencephalography (EEG).

3. Elevated cerebrospinal fluid globulin levels.

4. Elevated cerebrospinal fluid measles antibody titers.

5. Typical histological findings in brain biopsy or 


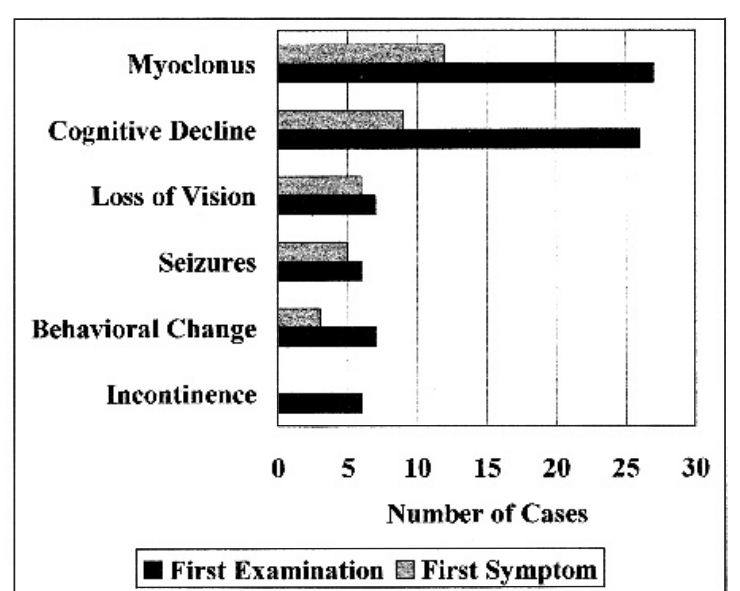

Figure 1. Clinical symptomatology.

autopsy.

The diagnosis was established if three out the five criteria were fulfilled. Those patients fulfilling the above criteria were selected and enrolled for the study. Their data was obtained under the following headings.

a. Preliminary data: name, age, sex, duration of symptoms, history of measles, history of measles vaccination.

b. Presence of symptoms like cognitive decline, myoclonic jerks, seizures, loss of vision, behavioral change, and sphincter dysfunction.

These patients were then evaluated clinically to look for abnormalities of higher mental functions, visual acuity, presence of chorioretinitis on fundus examination, presence of long tract signs and motor or sensory deficit. Clinical examination was followed by investigations including EEG, cerebrospinal fluid and serum examination to look for measles antibody titers. Neuroimaging, wherever possible, was carried out.

\section{Results}

A total of 32 cases were included in the study. Twenty four of these patients were males and eight were females. The age at presentation varied from 4 years to 21 years with a mean age of 13.4 years.

In our series 26 patients presented with cognitive decline, while myoclonus was seen in 27 patients (Figure 1). Almost $90 \%$ of the patients had at least one of these two symptoms. In the remainder, predominant seizures, loss of vision or behavioral change were the presenting symptoms. Six patients were incontinent by the time medical attention was obtained. It can thus be seen that cognitive decline and myoclonus were the essential features of the clinical symptomatology at the initial examination.

Figure 1 also depicts the symptoms at the onset of the disease. Relatives of two of our patients were unable to pinpoint the exact symptom amongst
Table 1. Uncommon clinical features at initial examination

\begin{tabular}{lc}
\hline \hline Features & Number of Cases \\
\hline Vision loss & 7 \\
Seizures & 6 \\
Behavioral change & 7 \\
Catatonia & 1 \\
\hline \hline
\end{tabular}

myoclonus and cognitive decline at the onset hence in these patients we considered that both symptoms began at the same time. Out of the 32 patients, features like vision loss, seizures, and behavioral changes were seen in 13 patients with one patient having a simultaneous onset of seizures with behavioral change (Table 1). Typical features like myoclonus and/or cognitive decline were seen in the remaining 19 patients. Thus in approximately $40.6 \%$ of patients uncommon features marked the onset of disease.

In our study nine patients were vaccinated for measles at nine months of age. The mean age at onset of the disease in the vaccinated group was 15.7 years as compared to 12.4 years in the unvaccinated group. The vaccinated group had a rapid clinical worsening as compared to the non-vaccinated group. The average duration of illness from the onset of first symptom to seeking medical attention was 3.2 months in the vaccinated group while it was 6.6 months in the non-vaccinated group. By using the Neurologic Disability Index, all the unvaccinated and six vaccinated patients were found to have subacute speed of progression, while three patients in the vaccinated group had acute evolution. 23 patients were unvaccinated. Relatives of 15 patients (65.2\%) could remember the presence of measles infection in the childhood, prior to the age of 4 years. The mean incubation period in these patients was at least 8.13 years. Out of the four patients with onset of illness after the age of 20 years three were vaccinated and, the single unvaccinated patient had suffered from measles at the age of 5 years.

Thirteen out of the 32 patients showed uncommon features at the onset of the disease (Figure 1). Amongst our series of 32 patients, seven patients presented with vision loss which was attributed to white matter lesions in the parieto-occipital region. Out of these seven, visual loss was the first symptom in six patients. Three other patients had signs of early optic atrophy but had no symptoms of loss of vision. Thus all patients in our series had cortical type of vision loss. Five (15.7\%) patients had onset with seizure disorder, while long tract signs in the form of spasticity which manifested as either hemiparesis or quadriparesis was seen in five $(15.7 \%)$ patients. The pyramidal tract signs were seen in later stages of the disease. The six patients 


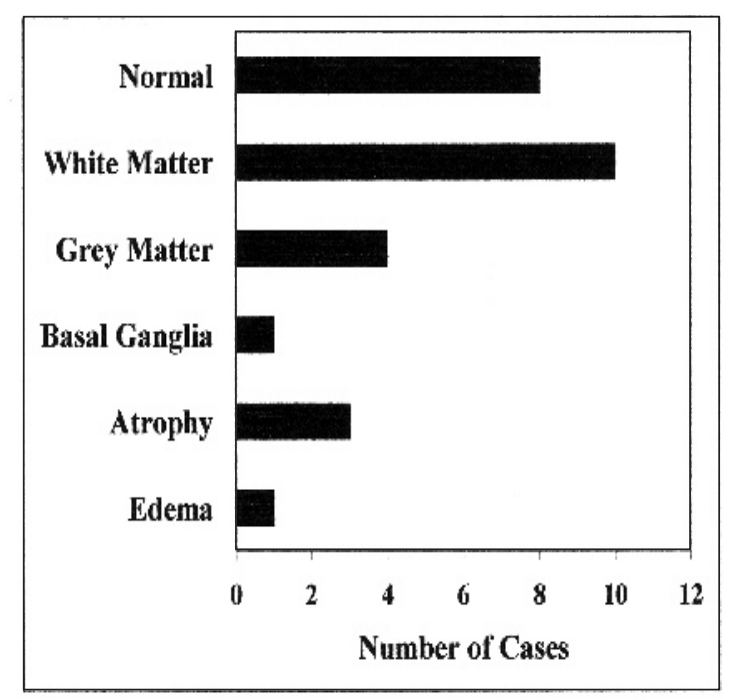

Figure 2. Imaging findings.

presenting with seizures had a prolonged history of seizures, with average duration of 15 months before cognitive decline set in. Seven patients had behavioural changes. It was the presenting symptom in three of them.

Twenty one patients were imaged in our series. Eighteen magnetic resonance imaging (MRI) and three computed tomography (CT) examinations were performed. Eight patients had normal imaging findings. Out of the remaining 13 patients abnormalities were seen in white matter, grey matter and basal ganglia. Changes were seen in parieto-occipital cortical and subcortical areas with symmetric involvement of the periventricular white matter in 10 out of 13 patients. Seven patients in this group of white matter lesions presented with vision loss. Three patients had involvement of white and grey matter together while isolated involvement of basal ganglia was seen in a single patient (Figure 2).

\section{Discussion}

SSPE is a chronic inflammatory disease of the central nervous system following childhood measles infection which is invariably fatal. The average age of presentation worldwide is between 5 and 15 years with the mean age being $9-10$ years $(1,2)$. The average age of patients in our study was 13.4 years, which is higher than other studies. This finding is in keeping with the fact that globally the average age of SSPE is increasing, which can be attributed to better vaccination coverage. In the present study, the vaccinated patients presented later than the unvaccinated ones by 3.3 years. There are some reports in which the average age has been shown to have decreased following vaccination; however the importance of this observation is unclear at present $(4,6)$.

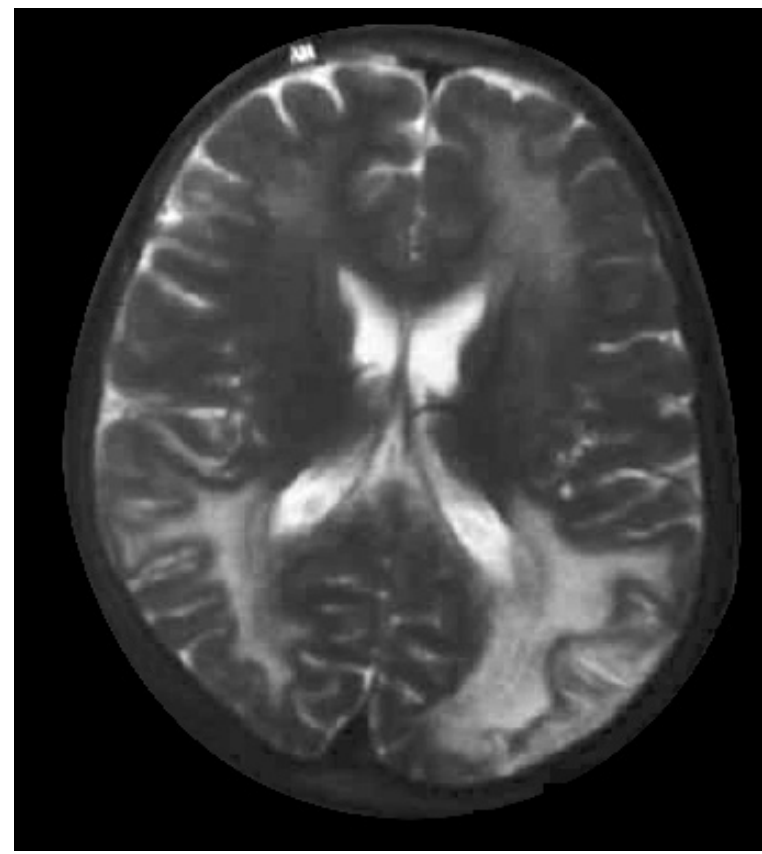

Figure 3. T2 weighted MRI showing increased signal in the parieto-occipital white matter and grey matter.

In our study, patients who had been vaccinated had more rapid course of disease, from the onset till the diagnosis, being 3.2 months as compared to 6.6 months in the unvaccinated group. Also, acute progression was seen only in the vaccinated patients, but we could not find any study to corroborate this finding. The association of measles vaccine and SSPE has been an issue of much importance. In patients of SSPE who had been vaccinated, It has been documented that the genomic structure of the measles virus from the vaccine did not match the virus obtained from the specimens of brain biopsy as reported by Jin et al. (8), and measles vaccination has not been causally associated with the development of SSPE. Our observation of rapid evolution of SSPE in vaccinated patients will need further scrutiny with larger number of patients.

The higher percentage of cases of SSPE amongst the vaccinees may be due to poor nutritional status of children in the developing countries resulting in poor uptake, or due to a different type of strain in the environment, subclinical measles infection prior to measles vaccination or due to faulty storage (cold chain) of the vaccine. We believe that one or more of these factors were operating in our vaccinated children who had SSPE.

Thirteen patients presented with unusual symptoms like loss of vision, seizures, or behavioral changes. Though loss of vision is a well documented symptom, studies have reported a higher incidence of visual loss amongst patients with adult onset SSPE with age ranging from 20-35 years $(2,9,10)$. However, in our study, the mean age of patients presenting with loss of vision was 14.8 years with 


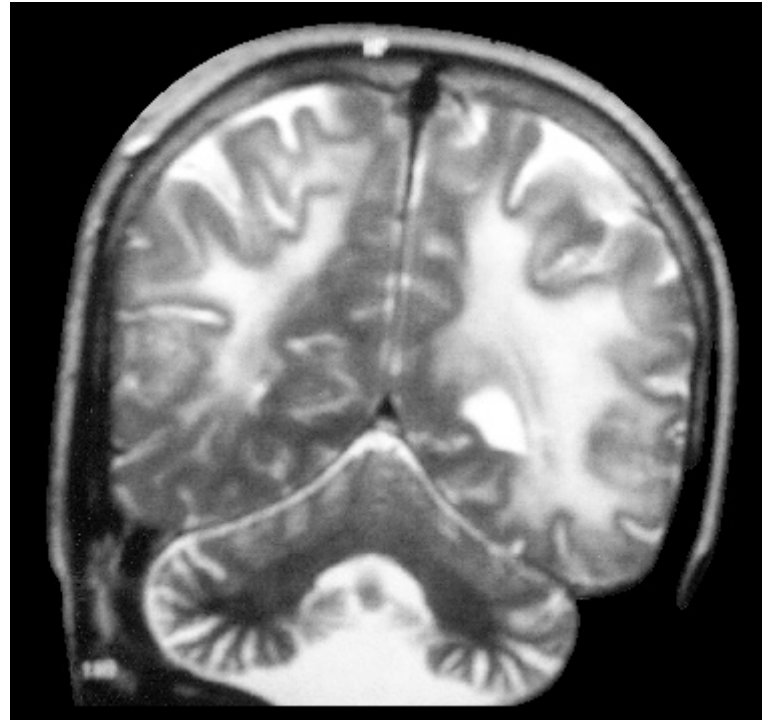

Figure 4. T2 weighted MRI showing increased signal in the parieto-occipital white matter and grey matter.

age range of 12-20 years. Visual loss was seen as frequently with the young as with the older patients. The vision loss in these patients was of the posterior type with neuroimaging features suggestive of diffuse white matter lesions in the parieto-occipital lobes which are best seen on the T2 weighted MRI images (Figure 3 and 4). Features suggestive of chorioretinitis were seen in four patients, out of which three patients also had early optic atrophy, however loss of vision was neither reported nor found in these four patients. Thus, at all ages, visual loss of the occipital type formed an important feature in the early phases of SSPE.

Those patients who presented with seizures had a longer duration of symptomatology till the diagnosis of SSPE. Most of these patients continued to experience seizures in spite of anti-epileptic medications and then they progressed slowly with evolution of myoclonus and cognitive decline as the time elapsed. All six patients experienced generalized seizures, out of which four patients began their illness with seizures alone and in the other two such seizures were either along with or following other symptoms respectively. Patients with "disease revealing" seizures, as seen in five of our patients, had a prolonged course of symptomatology, before cognitive decline set in. Kissani et al. (11) documented epilepsy in $30(42 \%)$ of their patients in a series of 70 patients. Among these 30 patients, "disease revealing" seizures were seen in $23 \%$ of patients. Özturk et al. (12) had seven (19.4\%) patients with seizures in their series of 36 patients. The incidence of seizures in our study is less as compared to studies mentioned in literature.

Wandering behavior was noted in four patients and it was the first symptom in two of them. These patients had uncontrolled urge to leave home
Table 2. Comparison of present and past study from our department

\begin{tabular}{lll}
\hline \hline & $\begin{array}{l}\text { Present study } \\
\mathbf{2 0 0 3}(\mathbf{n = 3 2}) \\
\mathbf{n ( \% )}\end{array}$ & $\begin{array}{l}\text { Singhal et al } \\
\mathbf{1 9 7 4}(\mathbf{n}=\mathbf{3 9}) \\
\mathbf{n}(\mathbf{\%})\end{array}$ \\
\hline Mean age (years) & 13.4 & 11.2 \\
Sex (male:female) & $24: 8$ & $36: 3$ \\
Myoclonus & $27(84.3)$ & $37(94.8)$ \\
Cognitive decline & $26(81.2)$ & $38(97.4)$ \\
Seizures & $6(18.7)$ & $13(33.3)$ \\
Hemiparesis & $5(15.7)$ & $2(5.1)$ \\
Chorioretinitis & $4(12.5)$ & $1(2.5)$ \\
Vision loss & $7(21.8)$ & $0(0)$ \\
\hline \hline
\end{tabular}

and would wander for hours before returning. Irritability and adamant behavior was seen in all the seven patients. Hyper religiosity was seen in one patient. He used to spend most of his awake hours in praying and worshipping. All the seven patients gradually developed more obvious cognitive decline and myoclonus over an average period of 5.9 months.

Out of 21 patients who underwent neuroimaging approximately one-third of patients had normal imaging. The most striking feature of neuroimaging was abnormalities in the white matter mainly involving the parieto-occipital cortical and subcortical regions. Changes were also detected in the grey matter and the basal ganglia but these changes were far and few as compared to the white matter changes. Early stages of SSPE shows edematous change or normal imaging while later stage of the disease shows marked atrophy of the brain which was seen in three of our patients (13). In our study there was no enhancement of lesions on CT scans, but Brismar et al. (14) have reported a case with rapid clinical deterioration and multiple areas of enhancement on neuroimaging.

A similar study regarding clinical aspects of SSPE was published from our department in 1974 (7). We compared the findings of our present study with the study done at our institution 30 years ago. As can be seen from Table 2, there has been no significant change in the mean age of patients. The striking preponderance of male patients continues, though less prominent. This is curious. Earlier it was believed that the social circumstances were related to this disparity. Those social issues have much changed over the past 30 years and this observation is difficult to explain and may be related to hormonal influence as suggested by Dyken et al (4). Zeman and Kolar (15) mention a male to female affection as 2.5:1 which is comparable to our study ratio of 3:1 (15). The majority of patients had myoclonus and cognitive decline. No case of vision loss was reported in the previous study. Hemiparesis, 
choreoathetosis, tremors, and generalized seizures were the other prominent features in the previous study out of which choreoathetosis and tremors were not seen in any of our patients.

\section{Conclusions}

SSPE is still an important cause of mental decline at young ages in our set up. The mean age of presentation has increased to 13.4 years, over the past 30 years. A proportion of patients had developed SSPE in spite of being vaccinated. Almost $40 \%$ of our patients presented with one or more of uncommon features like vision loss, seizures, and behavioral disturbances. These presentations need emphasis in the early diagnosis of this devastating condition.

\section{Acknowledgements}

We thank Dr. N.A. Mehta Shah, Professor and Head, Department of Neurology and Dr. G.B. Daver, Dean, Grant Medical College and Sir JJ group of Hospitals, Mumbai for their support and allowing us to present this work.

\section{References}

1. Dyken PR. Subacute sclerosing panencephalitis Current status. Neurol Clin 1985; 3: 179-196.

2. Garg RK. Subacute sclerosing panencephalitis. Postgrad Med J 2002; 78: 63-70.

3. Haddad FS, Risk WS, Jabbour JT. Subacute sclerosing panencephalitis in the Middle East: report of 99 cases. Ann Neurol 1977; 1: 211-217.

4. Dyken PR. Neuroprogressive disease of postinfectious origin: a review of resurging subacute sclerosing panencephalitis (SSPE). Ment Retard Dev Disabil Res Rev 2001; 7: 217-225.

5. Dyken PR, Cunningham SC, Ward LC. Changing character of subacute sclerosing panencephalitis in the United States. Pediatr Neurol 1989; 5: 339-341.

6. Anlar B, Köse G, Gürer $Y$, et al. Changing epidemiological features of subacute sclerosing panencephalitis. Infection 2001; 29: 192-195.

7. Singhal BS, Wadia NH, Vibhakar BB, Dastur DK. Subacute sclerosing panencephalitis 1-Clinical aspects. Neurol India 1974; 22: 87-94.

8. Jin L, Beard S, Hunjan R, Brown DW, Miller E. Characterization of measles virus strain causing SSPE: a study of 11 cases. J Neurovirol 2002; 8: 335 344.

9. Modlin JF, Halsey NA, Eddins DL, et al. Epidemiology of subacute sclerosing panencephalitis. J Pediatr 1979; 94: 231-236.

10. Singer C, Lang AE, Suchowersky O. Adult-onset subacute sclerosing panencephalitis: case reports and review of literature. Mov Disord 1997; 10: 342-353.

11. Kissani N, Ouzzani R, Belaidi H, Ouahabi H, Chkili T. Epileptic seizures and epilepsy in subacute sclerosing panencephalitis (report of 30 cases) Neurophysiol Clin 2001; 31: 398-405 (in French).

12. Özturk A, Gürses C, Baykan B, Gökyiğit A, Eraksoy M. Subacute sclerosing panencephalitis: clinical and magnetic resonance imaging evaluation of 36 patients. J Child Neurol 2002; 17: 25-29.

13. Alexander M, Singh S, Gnanamuthu C, Chandi S, Korah IP. Subacute sclerosing panencephalitis: $\mathrm{CT}$ and MR imaging in a rapidly progressive case. Neurology India 1999; 47: 304-307.

14. Brismar J, Gascon GG, von Steyern KV, Bohlega S. Subacute sclerosing panencephalitis: evaluation with CT and MR. AJNR Am J Neuroradiol 1996; 17: 761772 .

15. Zeman W, Kolar O. Reflections on etiology and pathogenesis of subacute sclerosing panencephalitis. Neurology 1968; 18: 1-7.

16. Mawrin C, Lins H, Koenig B, et al. Spatial and temporal disease progression of adult-onset subacute sclerosing panencephalitis. Neurology 2002; 58: 1568-1571.

17. Nakamura Y, Iinuma K, Oka E, Nihei K. Epidemiological features of subacute sclerosing panencephalitis from clinical data of patients receiving a public aid for treatment. No To Hattatsu 2003; 35: 316-320 (in Japanese).

18. Schneider-Schaulies J, Meulen V, SchneiderSchaulies S. Measles infection of the central nervous system. J Neurovirol 2003; 9: 247-252.

19. Mgone CS, Mgone JM, Takasu T, et al. Clinical presentation of subacute sclerosing panencephalitis in Papua New Guinea. Trop Med Int Health 2003; 8: 219-227.

20. Miki K, Komase K, Mgone CS, et al. Molecular analysis of measles virus genome derived from SSPE and acute measles patients in Papua, New Guinea. J Med Virol 2002; 68: 105-112. 